\title{
Differential Localization of Divalent Metal Transporter 1 with and without Iron Response Element in Rat PC12 and Sympathetic Neuronal Cells
}

\author{
Jerome A. Roth, ${ }^{1}$ Craig Horbinski, ${ }^{1}$ Li Feng, ${ }^{1}$ Kevin G. Dolan, ${ }^{2}$ Dennis Higgins, ${ }^{1}$ and Michael D. Garrick ${ }^{2}$ \\ Departments of ${ }^{1}$ Pharmacology and Toxicology and ${ }^{2}$ Biochemistry, State University of New York, Buffalo, New York 14214
}

\begin{abstract}
Two isoforms of divalent metal transporter 1 (DMT1) (Nramp2 and DCT1) are encoded by two mRNA species, one of which contains an iron response element (IRE) motif in the $3^{\prime}$ noncoding region. The subcellular distribution of the two isoforms of DMT1 is distinct, and the -IRE species accumulates in the nucleus of neuronal or neuronal-like cells. Reverse transcription-PCR and Western blot analysis of PC12 cells reveals that these cells express both forms of DMT1. Immunofluorescence and immunoblotting studies, using immunospecific antibodies to the -IRE form of DMT1, demonstrate that this form of the transporter, in PC12 cells, is predominantly localized in the nucleus, cell membrane, and neurites with only weak staining of the cell body. Studies using antibodies to the +IRE form indicate that this species of DMT1 is distributed within vesicles in the cell body and neurite projections, with minimal nuclear staining. Similar staining patterns are observed for the two forms of DMT1 in
\end{abstract}

cultures of sympathetic ganglion neurons isolated from perinatal rat pups. To determine whether nuclear localization of the -IRE form of DMT1 is constrained to neuronal or neuronal-like cells, immunocytochemical studies were performed with human embryonic kidney 293T (HEK293T), HEP2G hepatoma and medulIoblastoma, and rat Schwann cells. The -IRE-specific antibodies stained nuclei from medulloblastoma, whereas little nuclear staining was observed with HEK293T, hepatoma, or Schwann cells. The unexpected finding that the -IRE species of DMT1 selectively accumulates in the nucleus of neuronal and neuronallike cells leads us to postulate that the two proteins may have different functions in vivo.

Key words: divalent metal transporter 1; DMT1; Nramp2; iron transport; PC12 cells; sympathetic neurons; nuclear localization; transferrin receptor; HEK293T cells
Iron uptake in most mammalian cells occurs via the transferrin cycle. Iron is normally transported in the plasma in the ferric state by transferrin (Ponka, 1997). Transferrin subsequently binds the transferrin receptor on the cell surface, which then undergoes endocytosis, generating endosomal vesicles within the cell. The endosomes are acidified by a hydrogen ion ATPase pump, causing cooperative release of the metal from the transferrin-transferrin receptor (TfR) complex. Ferrous iron is then transported across the endosomal membrane via divalent metal transporter 1 (DMT1; Fleming et al., 1998). A proton gradient across the endosomal membrane is the driving force supporting metal ion-proton cotransport out of the endosome by DMT1 (Gunshin et al., 1997). DMT1 has a very broad substrate specificity and potentially transports other divalent cations, including $\mathrm{Mn}^{2+}, \mathrm{Cd}^{2+}, \mathrm{Zn}^{2+}, \mathrm{Co}^{2+}$, $\mathrm{Ni}^{2+}, \mathrm{Cu}^{2+}$, and $\mathrm{Pb}^{2+}$ (Gunshin et al., 1997).

Two isoforms of DMT1 present in mammalian cells result from alternate splicing of a single gene product (Gruenheid et al., 1995; Fleming et al., 1998; Lee et al., 1998). The two polypeptides share 543 residues on the $\mathrm{N}$-terminal end but differ primarily in the last 18 or $25 \mathrm{C}$-terminal residues. Only one of the two forms of mRNAs contains an iron response element (IRE) motif in the 3 '-noncoding region. Accordingly, we refer to these as -IRE and +IRE henceforth. Both isoforms contain 12 putative membrane-spanning domains and a consensus transport motif in the fourth intracellular loop (Gunshin et al., 1997). The presence of the IRE provides a site for binding of iron response proteins 1 and 2 (IRP1 and 2) (Rouault and Klausner, 1997; Haile, 1999; Schümann et al., 1999).

Received May 31, 2000; revised July 19, 2000; accepted July 27, 2000.

This work was funded by US Environmental Protection Agency Grant R26248010 (J.A.R.), National Science Foundation Grant BNS 8909373 (D.H.), and National Institutes of Health Grant HL48690 (M.D.G.). We thank Dr. Wade Sigurdson for expert technical assistance as the director of the Confocal Microscope and 3D Imaging Facility at the University at Buffalo.

Correspondence should be addressed to Dr. Jerome A. Roth, Department of Pharmacology and Toxicology, 102 Farber Hall, University at Buffalo, Buffalo, NY 14214. E-mail: jaroth@buffalo.edu.

Copyright (C) 2000 Society for Neuroscience $0270-6474 / 00 / 207595-07 \$ 15.00 / 0$
Binding of either IRP should stabilize DMT1 mRNA and increase expression of the + IRE protein. Recent studies demonstrate that IRP2 knock-out mice develop a progressive neurodegenerative disorder, including iron deposition in the CNS, similar to that observed in Parkinson's disease (Rouault, 1999). Thus, changes in the expression of proteins that are regulated by IRP1 and 2, which include DMT1 and TfR, may be responsible for the observed neurological disturbances observed in these knock-out mice.

Our laboratory has been characterizing the biochemical and molecular mechanisms responsible for heavy metal neurotoxicity and the contribution of transport in regulating these cytotoxic events. We have used rat PC12 cells as a model system to study manganese neurotoxicity, because these cells possess much of the biochemical machinery of dopaminergic neurons. Manganese can induce PC12 cell neuronal differentiation similar to that of nerve growth factor (NGF); however, it invariably leads to cell death (Lin et al., 1993). Recent studies suggest that cell death induced by manganese may be caused by either necrosis and/or apoptosis attributable to oxidative stress (Desole et al., 1996, 1997a,b; Hirata et al., 1998; Schrantz et al., 1999; Roth et al., 2000). Manganese has been reported to be taken up into PC12 cells by a transport system with characteristics resembling those of DMT1 (Kim et al., 1993), although the significance of the resemblance has been questioned (Yanagiya et al., 2000). Thus, studies were performed to determine whether DMT1 is present in PC12 cells to participate in manganese uptake. Our results reveal that PC12 cells contain both species of DMT1 and that the two transporters are distributed in different cellular compartments within both PC12 cells and cells of neuronal origin. In addition, the two isoforms of DMT1 only partially colocalized with TfR.

\section{MATERIALS AND METHODS}

Cell culture. PC12 cells were maintained in DMEM (Life Technologies, Grand Island, NY) supplemented with 10\% FBS (HyClone, Logan, UT), $5 \%$ horse serum (JRH Biosciences, Lenexa, KS), $100 \mathrm{U} / \mathrm{ml}$ penicillin, and $100 \mu \mathrm{g} / \mathrm{ml}$ streptomycin (Lin et al., 1993). Cells were grown at $36.5^{\circ} \mathrm{C}$ in a 
humidified atmosphere containing $5 \% \mathrm{CO}_{2}$ as described previously (Lin et al., 1993) and subcultured every $2-3 \mathrm{~d}$ at $\sim 70-80 \%$ confluency.

Antibodies to $+I R E$ and $-I R E$ forms of DMT1. To characterize the subcellular distribution of the two species of DMT1, it was necessary to produce antibodies that can selectively recognize each specific isoform (Gunshin et al., 1997; Fleming et al., 1998). Accordingly, peptides corresponding to the $\mathrm{C}$-terminal sequence of each protein were synthesized for preparation of antibodies. Antibodies to the two forms of DMT1 were prepared by $\alpha$ Diagnostic International Inc. (San Antonio, TX) from the following C-terminal peptides: +IRE peptide (545-561), SISKVLLSEDTSGGNTK; and -IRE peptide (547-568), GLTARPEIYLLNTVDAVSLVSR. These peptides were chosen on the basis of their representing the most structurally distinct sequences of the two isoforms of the transporter and an evaluation of their potential immunogenicity. The peptides were separately attached to keyhole limpet hemocyanin, and each was injected into two rabbits. The antisera were recovered, and the antibodies were immunoaffinity-purified against the respective peptides attached to Sepharose. Titers were determined against the immunizing peptides by ELISA and were used as a guide in selecting dilutions for Western blotting and immunofluorescent microscopy described below.

Identification of the +IRE and -IRE forms of DMT1 in PC12 cells by reverse transcription-PCR. Forward and reverse primers for + IRE DMT1 and -IRE DMT1 were used selectively to assess PCR products for the + IRE and -IRE forms of DMT1 in PC12 cells and cultured rat sympathetic neurons. For the +IRE form the forward primer sequence was 5'-CGGTAAGCATCTCTAAAG, representing bp 1732-1750, and the reverse primer was 5'-TAGCAGCATGCTATTTGAC, representing bp 1980-1998. To assess PCR products for the -IRE forms of DMT1, the forward primer sequence was 5'-TCTAGATGACCAACAGCC, representing bp 1699-1716, and the reverse primer was 5'-GCAGACACAAGCCTGCGT, representing bp 1945-1962. Both selections are in the 3 ' untranslated region. The reverse transcription-PCR reaction ( 25 cycles) was performed, and the resulting +IRE DMT1 and -IRE DMT1 PCR products, 267 and $264 \mathrm{bp}$, respectively identified on agarose gels, were each ligated into the pCR2.1-TOPO vector and transformed into Escherichia coli. Colonies containing the +IRE DMT1 or -IRE DMT1 inserts were selected with ampicillin and identified by their white color. These colonies were grown overnight in Luria-Bertani broth containing ampicillin, and the plasmid DNA was isolated, digested with EcoRI, and resolved on 1\% agarose gels to confirm the presence and size of the inserts. The plasmid DNA containing the expected size insert was sent to the Center for Advanced Molecular Biology and Immunology, State University of New York at Buffalo, for DNA sequence analysis, and the results were compared with the sequence from GenBank (accession numbers AF008439 and AF029757)

Identification of the +IRE and -IRE forms of DMT1 in PC12 cells by Western blot analysis. Western blot experiments were performed to determine the protein expression of DMT1 in PC12 cells. Whole-cell lysates and isolated nuclei were prepared, and $60 \mu \mathrm{g}$ of protein from each sample was subjected to SDS-PAGE on a $12 \%$ acrylamide gel. Nuclei were isolated by differential centrifugation on a discontinuous sucrose gradient according to the method of Cassano et al. (1996). The proteins were transferred to an Immobilon-P membrane and were subsequently treated with affinitypurified antibodies to either the +IRE or -IRE form of DMT1. DMT1 was detected using horseradish peroxidase-conjugated secondary antibody for rabbit IgG with Pierce (Rockford, IL) SuperSignal chemiluminescent substrate.

Isolation and culture of sympathetic neurons. Sympathetic neurons from perinatal Holtzman rat pups (Harlan Sprague Dawley, Indianapolis, IN) were isolated and maintained in culture as described previously (Higgins et al., 1991). Dissociated cells were plated onto poly-D-lysine-coated (100 $\mu \mathrm{g} / \mathrm{ml})$ coverslips at a density of $\sim 10$ cells $/ \mathrm{mm}^{2}$ and maintained in serumfree medium containing $\beta$ NGF $(100 \mu \mathrm{g} / \mathrm{ml})$ and human transferrin $(20$ $\mu \mathrm{g} / \mathrm{ml}$ ). Non-neuronal cells (i.e., fibroblasts and Schwann cells) were eliminated by adding the antimitotic agent cytosine-D-arabinofuranoside for 48 hr. Bone morphogenic protein 7 (BMP7; $50 \mu \mathrm{g} / \mathrm{ml}$ ) was then added to induce dendritic outgrowth (Lein et al., 1995). DMT1 in these cells was detected immunocytochemically with the two antibodies to DMT1. In some experiments, the antimitotic agent was not added to compare the subcellular distributions of DMT1 in Schwann cells to that in neurons.

Ectopic expression of DMT1. Ectopic expression of rat -IRE DMT1 in human embryonic kidney 293T (HEK293T) cells followed the method of Fleming et al. (1998) as modified by Garrick and Dolan (2000). Briefly, HEK293T cells were grown in DMEM with $10 \% \mathrm{FBS}$ at $37^{\circ} \mathrm{C}$ in $95 \% \mathrm{O}$, and $5 \% \mathrm{CO}_{2}$ in a $\mathrm{CO}_{2}$ incubator. Transfections were performed with DMRIE-C according to the manufacturer's instructions (Life Technologies, Rockville, MD). Cells transfected with $0.5 \mu \mathrm{g}$ of the pMT2-DMT1 expression plasmid in $1 \mathrm{ml}$ of media were examined $\sim 48 \mathrm{hr}$ later.

Immunocytochemistry. Cell cultures were fixed in $4 \%$ paraformaldehyde in $0.1 \mathrm{~mm}$ phosphate buffer and permeabilized for $3 \mathrm{~min}$ with $0.1 \%$ Triton $\mathrm{X}-100$ in PBS $(0.15 \mathrm{M} \mathrm{NaCl}$ and $0.01 \mathrm{~m}$ sodium phosphate buffer, $\mathrm{pH} 7.3)$. The two forms of DMT1 were localized by indirect immunofluorescence using previously described procedures of Higgins et al. (1991). Affinitypurified polyclonal rabbit antiserum that specifically cross-reacts with either the +IRE or -IRE species of the transporter was used as the primary antibody, and rhodamine-conjugated goat anti-rabbit IgG (Boehringer Mannheim, Indianapolis, IN) or Alexa-594 goat anti-rabbit IgG

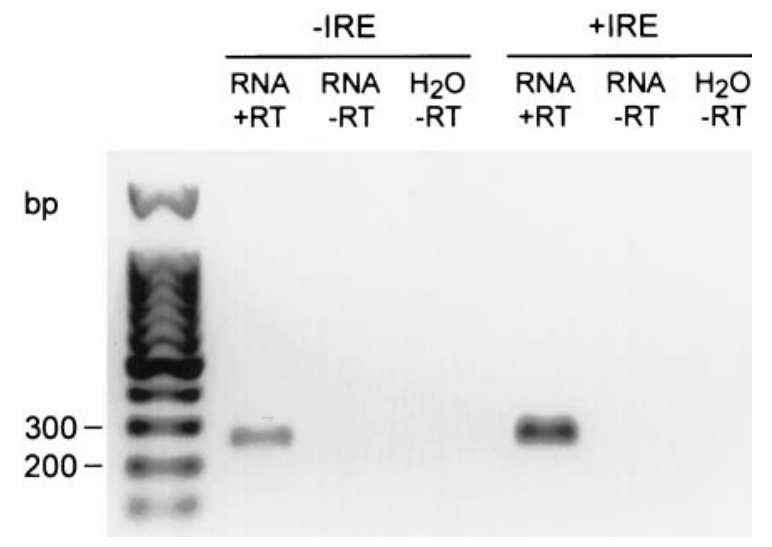

Figure 1. RT-PCR products of the +IRE and -IRE species of DMT1 from PC12 cells. The two bands identified on the gel correspond to the expected oligonucleotide lengths based on the primers used for each isomer of DMT1.

(Molecular Probes, Eugene, OR) for the HEK293T cells was used as the secondary antibody. In some experiments OX-26 (murine monoclonal anti-rat transferrin receptor; Serotec, Raleigh, NC) was used as a second primary antibody, and Alexa-488 goat anti-mouse IgG (Molecular Probes) was used as the secondary antibody. Immunostained cultures were mounted in Elvanol (DuPont, Wilmington, DE) and analyzed by conventional fluorescence (Optiphot; Nikon, Melville, NY) and confocal laser microscopy (Bio-Rad, Hercules, CA; 1024 confocal with a krypton-argon laser linked to a Nikon Optiphot microscope). Images were obtained using a $60 \times$ lens, numerical aperture (NA) 1.4, except for Figure 4, for which a $20 \times$ lens, NA 0.75 , was used.

\section{RESULTS}

\section{Identification of DMT1 mRNA and protein in PC12 cells}

Initial studies were performed to determine whether PC12 cells express DMT1 mRNA isoforms. As illustrated in Figure 1, reverse transcription (RT)-PCR products from the appropriate - IRE and + IRE primers each yielded a single major band, consistent with the expected number of base pairs for both forms of DMT1. The nucleotide sequence of the two PCR products was analyzed and found to correspond to the known sequences for the -IRE and + IRE isoforms of DMT1. Using the cloned PCR products as probes, Northern blots also confirmed the presence of both species of DMT1 in PC12 cells (data not shown). Western blots detected the expression of the +IRE and -IRE protein in these cells (Fig. 2 ). A single major band was observed with both antibodies, with an $M_{\mathrm{r}}$ of $\sim 65 \mathrm{kDa}$, in good agreement with the expected molecular weight based on the amino acid sequences for both isoforms of DMT1.

\section{Subcellular localization of +IRE and -IRE forms of DMT1 in PC12 cells}

The subcellular distribution of the two isoforms of DMT1 was examined to help understand their function in iron and divalent metal transport. Both forms of DMT1 are proteins with 12 putative membrane-spanning domains that are presumed to be located at the cell membrane and/or within the endosomal fraction of the cell. This expectation is supported by previous immunocytochemical studies using antibody preparations that are unable to fully distinguish the two species of the transporter (Su et al., 1998; CanonneHergaux et al., 1999; Gruenheid et al., 1999; Tabuchi et al., 2000; Trinder et al., 2000). Accordingly, immunocytochemical studies were performed with PC12 cells treated with NGF for $5 \mathrm{~d}$ using affinity-purified antibodies specific for each form of DMT1. Confocal microscopy images demonstrate that the +IRE form of DMT1 is mainly distributed in a punctate manner throughout the cell body with little if any staining of the nucleus (Fig. $3 B, D$ ). The pattern of staining within the cell body is rather diffuse with relatively little staining associated with the plasma membrane. Although somewhat difficult to see in the optical sections used in these figures, there is also intense staining within the neurites of 


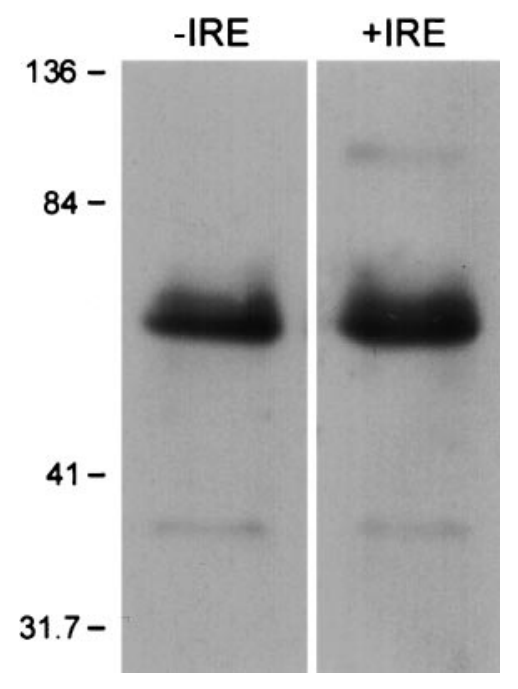

Figure 2. Western blots of the -IRE and +IRE species of DMT1 from PC12 cells. Western blots were performed on protein isolated from PC12 cells as described in Materials and Methods. The $M_{\mathrm{r}}$ of $\sim 65 \mathrm{kDa}$ for the two bands appearing on the gel corresponds to the expected size of the two isoforms of DMT1 based on the known amino acid composition.
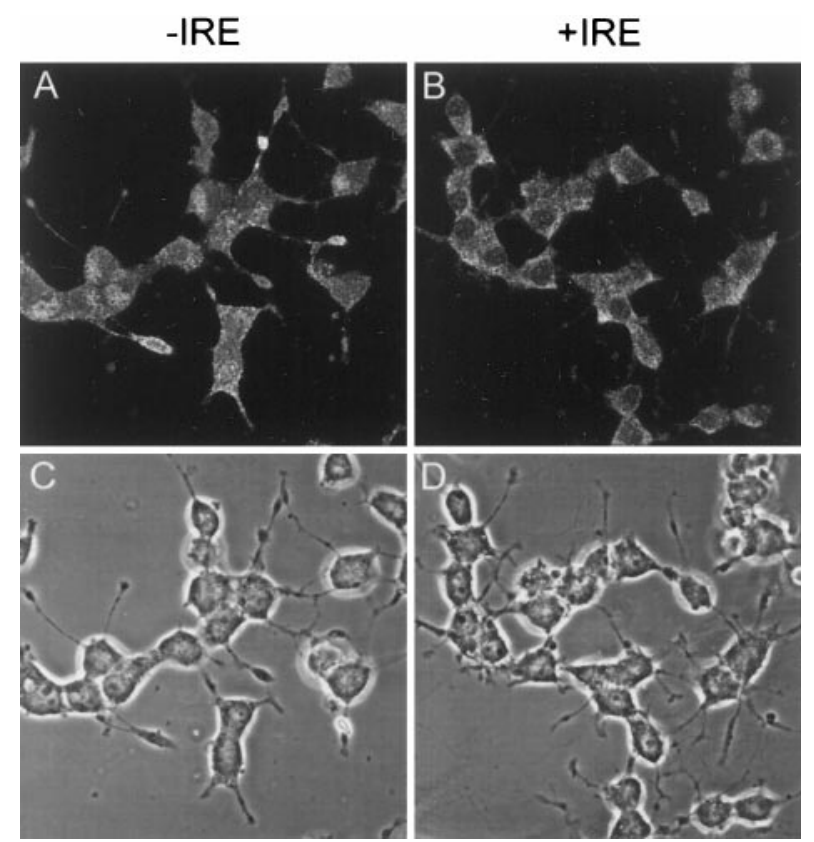

Figure 3. Confocal fluorescence micrographs of PC12 cells immunostained with antibodies specific for the $-\operatorname{IRE}(A)$ and $+\operatorname{IRE}(B)$ species of DMT1, along with the corresponding phase-contrast images. Cells were exposed to NGF for $5 \mathrm{~d}$ before they were immunostained.

NGF-treated PC12 cells. In contrast, the pattern of staining with the -IRE antibody in PC12 cells is considerably different from that observed with the +IRE antibody preparation (Fig. 3A,C). Most striking is the intense staining that is present in both the nucleus and the plasma membrane. A minimum of $80 \%$ of the PC12 cells in the presence of NGF display nuclear staining. A similar pattern of staining was also obtained in control cells not treated with NGF. In both cases, there is an absence of staining in the nucleolus and only weak and diff use staining of the cell body. Although not shown, the intensity of nuclear staining appeared to be enhanced during longer culture times, and this response was independent of exposure to NGF. As shown in Figure $3 A$, there was also strong -IRE staining of the cytoplasmic fraction within the neurites and growth cones in cells treated with NGF.

Western blots were also performed to verify the selective stain-

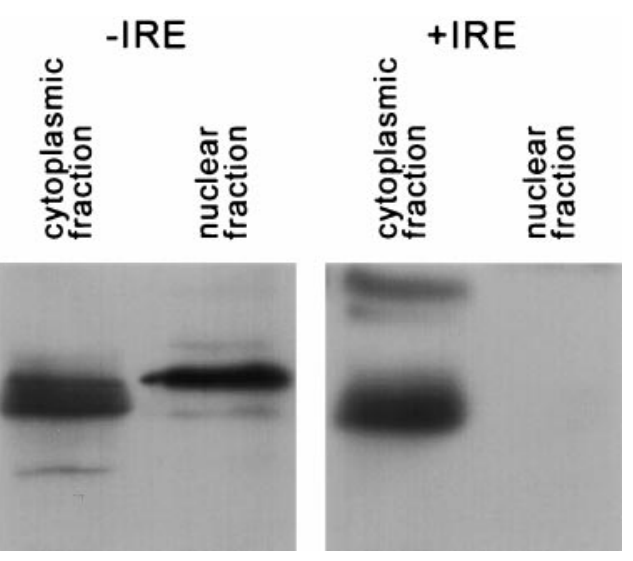

Figure 4. Western blots of subcellular fractions to detect the -IRE and + IRE sequences of DMT1. The +IRE species contains a major band at $\sim 65 \mathrm{kDa}$ and a diffuse band at $\sim 91 \mathrm{kDa}$ in the cytoplasmic cell fraction (cytosolic and membrane fraction) and no staining in the nuclear fraction. The -IRE species is detected at $\sim 65 \mathrm{kDa}$ in the cytoplasmic fraction and at $\sim 67 \mathrm{kDa}$ in the nuclear fraction.

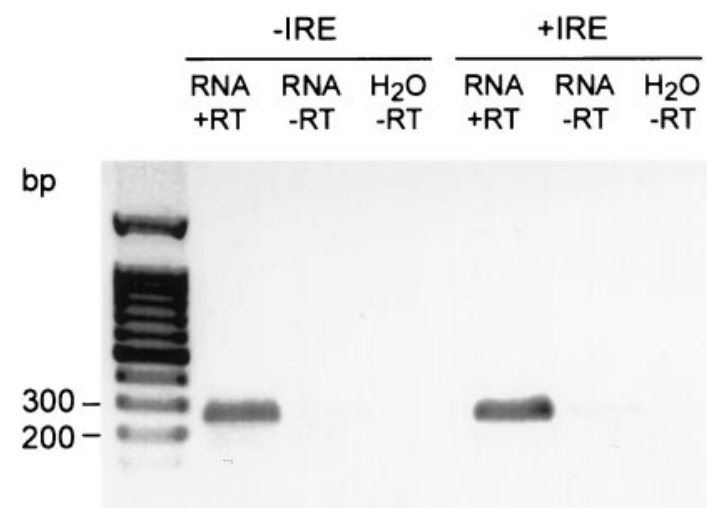

Figure 5. RT-PCR products of the +IRE and -IRE species of DMT1 from cultured rat sympathetic neurons treated for $5 \mathrm{~d}$ with BMP7. The two bands identified on the gel correspond to the expected oligonucleotide size based on the primers used for each isomer of DMT1.

ing pattern of the two forms of DMT1 in isolated nuclei from PC12 cells. As illustrated in Figure 4, a strong band at slightly higher than the expected $65 \mathrm{kDa}$ appeared with the isolated nuclear fraction when immunostained with antibody for the -IRE form of DMT1. In contrast, antibody to the +IRE species failed to detect a band with the nuclear fraction on the same gels. However, both antibodies recognized a strong band at $\sim 65 \mathrm{kDa}$ with the remaining cell fraction.

\section{Subcellular localization of + IRE and -IRE forms of DMT1 in rat sympathetic neurons}

Previous studies indicated there was no nuclear staining of DMT1 in rat duodenal enterocytes using an antibody that reacted with a common epitope to both forms of the transporter (Trinder et al., 2000). On the basis of these findings, we decided to determine whether the nuclear staining observed with the -IRE form of DMT1 in PC12 cells is present in other neuronal cells. RT-PCR experiments reveal that sympathetic neurons isolated from perinatal rat pups express DMT1 mRNA. A single band appeared for both the +IRE and -IRE isoforms of DMT1 corresponding to the expected base size (Fig. 5).

When the subcellular distribution for both species of DMT1 was determined in these sympathetic neuronal cells, a pattern of staining similar to that observed with PC12 cells was obtained (Fig. 6). Selected optical sections from two neurons using antibodies directed against the -IRE form of DMT1 demonstrate intense staining of both the nucleus and cell membrane but not the nucle- 
-IRE
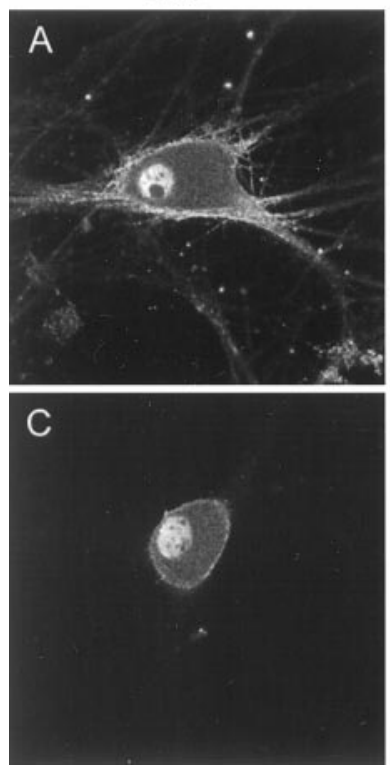

+ IRE
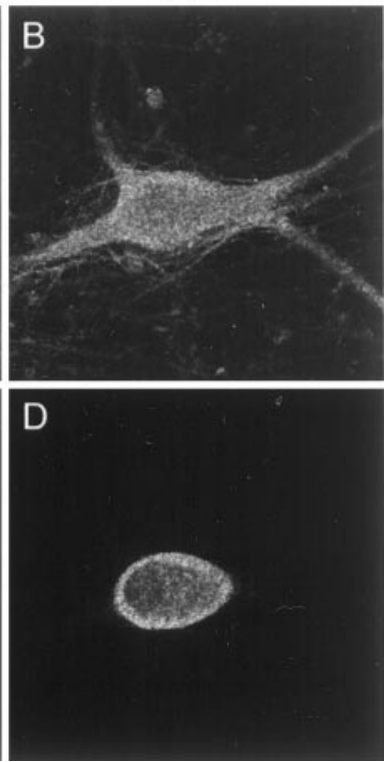

Figure 6. Confocal fluorescence micrographs of cultured rat sympathetic neurons treated for $5 \mathrm{~d}$ with BMP7 $(50 \mu \mathrm{g} / \mathrm{ml})$ immunostained with antibodies specific for the $-\operatorname{IRE}(A, C)$ and $+\operatorname{IRE}(B, D)$ species of DMT1. In the two optical sections $(A, B)$, near the substratum on which the cells were grown, neurites are visible; in sections $C$ and $D$, which are well above the substratum, staining patterns of the nucleus are most visible.

olus of sympathetic neurons treated with BMP7 (Fig. 6A,C), and there was relatively weak staining of the cell body compared with that of the nucleus. In contrast, reactions using antibodies against the +IRE form of DMT1 (Fig. 6B,D) resulted in a punctate and diffuse staining pattern in the cell body, including the cell membranes, with essentially total absence of staining of the nucleus. Both antibody preparations were capable of staining axonal and dendritic projections of the BMP7-treated sympathetic neurons (Fig. 6A,B).

To demonstrate that the staining pattern observed in the sympathetic neurons was dependent on the specificity of the antibodies, studies were performed to ascertain whether the two peptides used for production of the antibodies could inhibit their respective immune responses. As illustrated in Figure 7, $A$ and $B$, the specific peptide from the -IRE species greatly attenuated the response with the -IRE antibody, demonstrating that the antibody is reacting with the expected DMT1 epitope. As shown in Figure 7, $C$ and $D$, a similar result was observed when the peptide specific for the +IRE form of DMT1 was used to inhibit the +IRE-specific antibody response. As an additional control to verify specificity of the responses, studies were also performed to determine whether the + IRE peptide could inhibit the staining of the -IRE antibody and whether the -IRE peptide could inhibit that of the +IRE antibody (data not shown). As expected, there was no diminution in signal strength when the peptides were used in this manner, further confirming the specificity of the antibody preparations.

\section{Isoforms of DMT1 minimally colocalize with transferrin receptors in sympathetic neurons}

TfR serves as a means of tracking intracellular iron metabolism and endocytosis, having already been well characterized in many types of cells. Therefore, experiments were performed to determine whether either species of DMT1 colocalizes with TfR, which has previously been reported to be primarily restricted to the cell body and dendrites of cultured neurons (Cameron et al., 1991). Figure 8, $A$ and $C$, illustrates selected optical sections for TfR and the -IRE form of DMT1, and Figure $8, B$ and $D$, shows sections for TfR and the +IRE form of DMT1. TfRs are also found preferentially in perinuclear vesicles and in the region of the cell surface with total absence of staining of the nucleus (Fig. 8C,D).
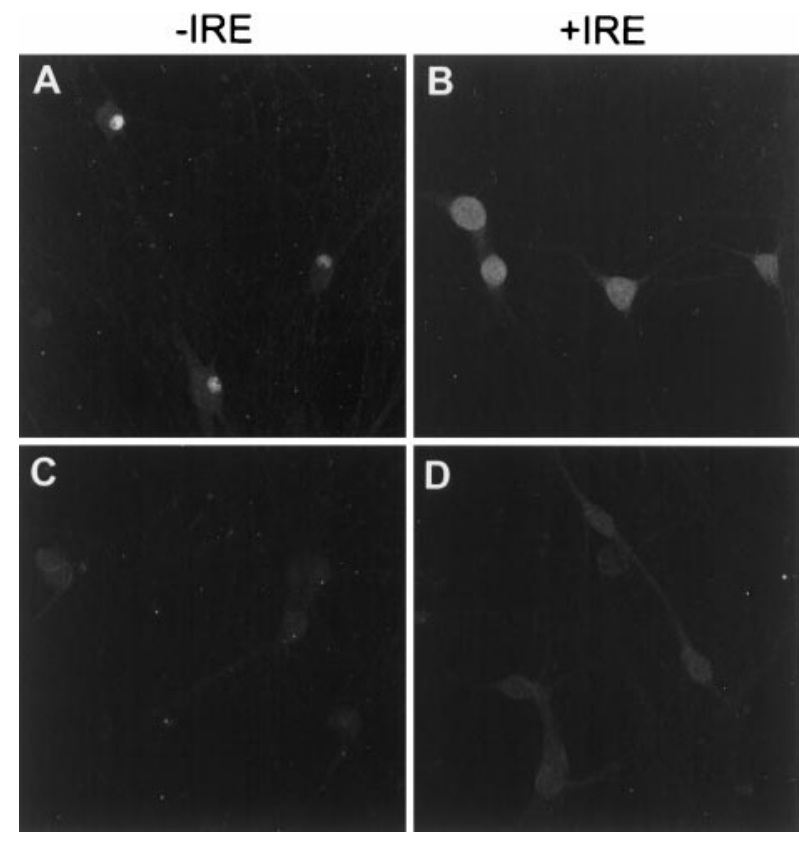

Figure 7. Confocal fluorescence micrographs of cultured rat sympathetic neurons treated for $5 \mathrm{~d}$ with BMP7 immunostained with antibodies specific for the $-\operatorname{IRE}(A, C)$ and $+\operatorname{IRE}(B, D)$ species of DMT1. Peptides to the $\mathrm{C}$-terminal sequence, used to generate the antibodies, for either the -IRE $(C)$ or +IRE $(D)$ forms of DMT1 were added before immunostaining. In both cases, addition of peptides attenuated the intensity of the immunostaining of the neurons.
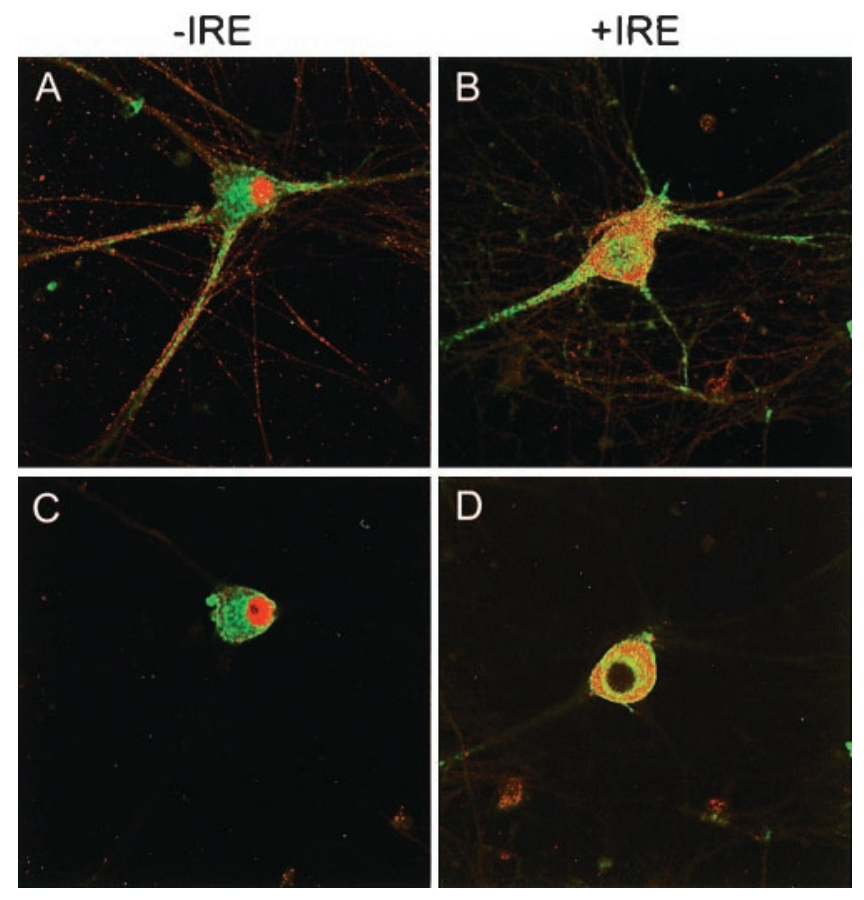

Figure 8. Confocal fluorescence micrographs of cultured rat sympathetic neurons treated for $5 \mathrm{~d}$ with BMP7 immunostained with antibodies specific for the $-\operatorname{IRE}(A, C)$ and $+\operatorname{IRE}(B, D)$ species of DMT1 and TfR. Green and red denote TfR and DMT1, respectively. Orange and yellow patches represent areas in which TfR and DMT1 are colocalized. In the two optical sections $(A, B)$, near the substratum on which the cells were grown, neurites are visible; in $C$ and $D$, which are well above the substratum, staining patterns of the nucleus are most visible.

Antibodies to TfR also strongly stain dendrites of the sympathetic neurons treated with BMP7 (Fig. $8 A, B$ ). A strikingly different picture emerges when the staining pattern for the -IRE isoform of DMT1 is compared with that for TfR (Fig. 8A,C). The two 

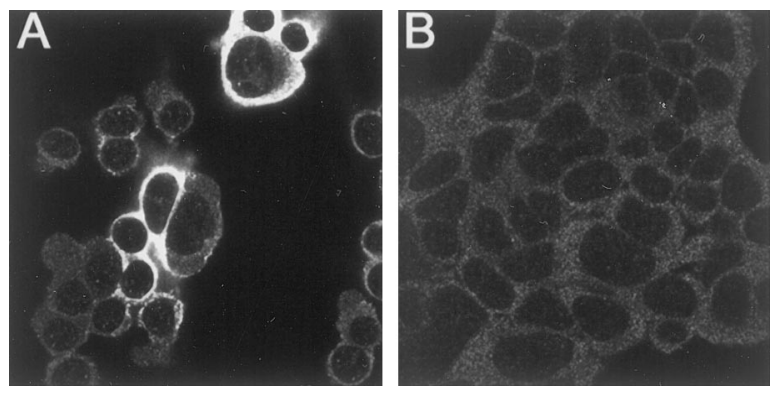

Figure 9. Confocal fluorescent micrographs of cultured HEK293T cells transfected with a construct of -IRE DMT1 immunostained with antibodies specific for the $-\operatorname{IRE}(A)$ and $+\operatorname{IRE}(B)$ species of DMT1. The more heavily stained cells seen with the -IRE antibody correspond to cells successfully transfected with the -IRE form of DMT1. No nuclear staining was observed in all cells, including those overexpressing the -IRE isoform of DMT1.

proteins only partially colocalize on the cell surface, with the most intense staining of the -IRE species of DMT1 observed in the nucleus. Considerably less staining of this form of DMT1 is observed in the cytosol of the cell body; rather, it is present on the cell surface and the surface of the dendrites, appearing in the dendrite extensions well away from the cell body in a punctate manner. The staining pattern for the -IRE isoform of DMT1 is minimally present in perinuclear vesicles, whereas staining for $\mathrm{TfR}$ is predominantly perinuclear as expected. TfR staining is also relatively more intense both in the cell surface and within the cell body than that for -IRE protein, and, as noted above, TfR staining is totally absent from the nucleus. TfR also appears to be present on the cell surface independent of this isoform of the transporter. The staining pattern for the +IRE isoform of DMT1 and TfR (Fig. 8B,D) also reveals both different and similar localization patterns for these proteins. TfR staining only partially colocalized with that of the +IRE isoform within the cell body, and staining of TfR within the dendrites is more intense. As illustrated in Figure 8, $B$ and $D$, and as noted above, the +IRE form of DMT1 is found in vesicles distributed relatively uniformly within the cytosol and infrequently distributed on the cell surface. TfR also appears to be present on the cell surface without the +IRE transporter. Comparison of TfR to the +IRE isoform of DMT1 in sections where the nucleus is visible (Fig. $8 B$ ) again reveals that neither antibody stains the nucleus.

\section{Subcellular localization of +IRE and -IRE forms of DMT1 in HEK293T cells}

Because enterocytes were reported to lack nuclear staining for DMT1 (Trinder et al., 2000), studies were also performed to determine the subcellular distribution of these transporters in other non-neuronal cell preparations. These studies also serve as a means of further testing the specificity of these antibodies and determining whether they cross-react with human DMT1 isoforms. Initial studies with HEK293T cells that were transfected with the -IRE construct of DMT1 demonstrate that the -IRE-specific antibody is capable of cross-reacting with human DMT1 (Fig. 9). Untransfected cells exhibited a relatively low level of expression of this form of DMT1. Approximately $20 \%$ of the cells were successfully transfected, as indicated by a large increase in immunostaining with the -IRE form of DMT1. Unlike the observed distribution pattern seen with the neuronal cell lines described above, staining exclusively manifested only in vesicles within the cell body and/or on the cell membrane, with a total absence of nuclear staining. The staining pattern for the +IRE species of nontransfected HEK293T cells was also weak (Fig. 9B) and, as anticipated, did not change in cells transfected with the -IRE construct of DMT1. These results verify the specificity of both the +IRE and -IRE antisera and their ability to cross-react selectively with the two isomers of human DMT1 and demonstrate the absence of
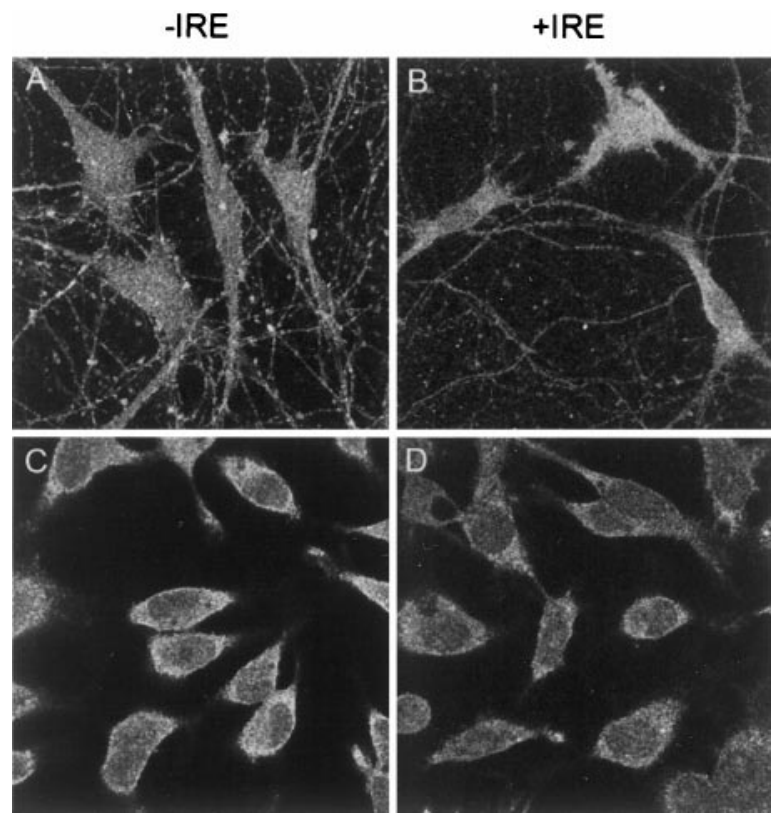

Figure 10. Confocal fluorescent micrographs of rat Schwann cells $(A, B)$ and human HEP2G cells $(C, D)$ immunostained with antibodies specific for the $-\operatorname{IRE}(A, C)$ and $+\operatorname{IRE}(B, D)$ species of DMT1.

nuclear staining of the -IRE species in HEK293T cells even when ectopically overexpressed.

\section{Subcellular localization of +IRE and -IRE forms of DMT1 in other cultured cells}

The subcellular distribution of the two forms of DMT1 was also assessed in rat Schwann cells that are present when rat sympathetic neurons are grown in the absence of an antimitotic agent. As illustrated in Figure 10, staining of the -IRE species of the transporter in rat Schwann cells was rather diffuse and evenly distributed throughout the cell in both the cytoplasm and nucleus, and unlike in the sympathetic neuronal cultures, there was no excess accumulation of this form of DMT1 in the nucleus. The absence of the antimitotic agent in the cultures does not account for the lack of nuclear staining of the Schwann cells, because neuronal cells cocultured in the presence of these cells exhibited intense nuclear staining (results not shown). As illustrated in Figure $8 B$, staining of the +IRE form of DMT1 in Schwann cells was also diffuse throughout the cell body, although there was clearly a decreased signal in the nucleus of these cells. In addition, we examined the -IRE and +IRE DMT1 staining pattern in several human cell lines, including HEP2G hepatoma cells (Fig. 10C,D) and medulloblastoma cells (Fig. 11A,B), to determine whether the antibody preparations would display the same subcellular distribution of DMT1 in non-neuronal and neuronal cells from human tissues. There was diff use cytoplasmic staining of both the +IRE and -IRE species of DMT1 in the HEP2G cells, but no nuclear staining was observed. In contrast, only the -IRE isoform of DMT1 of human medulloblastoma cells displayed nuclear staining (Fig. 11, compare $A, B$ ). The phase-contrast images (Fig. 11C,D) reveal that the nuclei of these cells are extremely large and represent a major portion of the cell volume. These data reaffirm that the two antibody preparations are capable of selectively recognizing appropriate epitopes on the +IRE and -IRE forms of human DMT1 and again support the previous data that the -IRE species of this transporter preferentially distributes to the nucleus in neuronal and neuronal-like cells. This is further supported by studies demonstrating that the -IRE species is present in the nucleus of rat dorsal root ganglion cells but is absent in isolated pancreatic islet cells (data not shown). 
-IRE
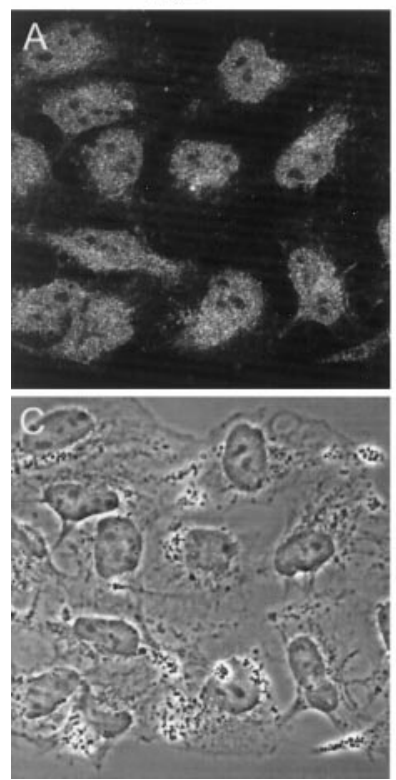

+IRE
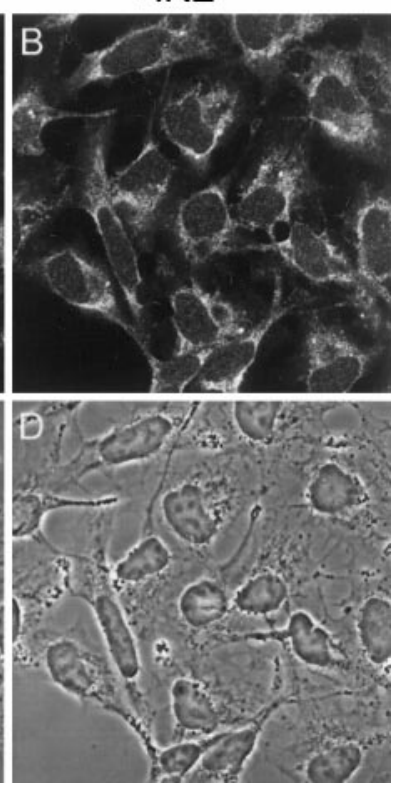

Figure 11. Confocal fluorescent micrographs of human medulloblastoma cells immunostained with antibodies specific for the $-\operatorname{IRE}(A)$ and $+\operatorname{IRE}$ $(B)$ species of DMT1 along with the corresponding phase-contrast images $(C, D)$.

\section{DISCUSSION}

We have previously shown that exposure of PC12 cells to manganese causes cell death in a time- and concentration-dependent manner (Roth et al., 2000). Our finding that cell death is associated with a decrease in ATP levels implies that intracellular levels of manganese may be mediating toxicity. Thus, the mechanism by which manganese is transported into PC12 cells becomes of prime importance, and our initial studies focused on determining whether these cells contain one or both isoforms of DMT1. Results from RT-PCR, immunoblots, and immunocytochemical studies confirm the presence of both forms of the transporter in PC12 cells. Using affinity-purified antibody preparations selective for each form of DMT1, we demonstrated that both species of the transporter possess a molecular weight of $\sim 65 \mathrm{kDa}$, in agreement with the expected value based on the known amino acid sequence for the two proteins. A range of values from 52 to $90-116 \mathrm{kDa}$ has been previously reported (Su et al., 1998; Canonne-Hergaux et al., 1999; Gruenheid et al., 1999; Tabuchi et al., 2000; Trinder et al., 2000). The differences probably reflect variations in source, preparation, glycosylation, and means of detection. Our size matches that found by Tabuchi et al. (2000) in COS-7 cells.

Immunocytochemical experiments clearly demonstrate that the two proteins are distributed in different subcellular compartments. The accumulation of the -IRE isoform in the nucleus of neuronal cells is the most surprising finding, whereas the +IRE form was present on the cell membrane and dispersed in a punctate manner within the cell but absent from the nucleus. A similar staining pattern was observed with cultured rat sympathetic neurons. Immunoblot studies are consistent with this in that only the -IRE species is present in isolated nuclei of PC12 cells. Consistent with this is the recent finding demonstrating the nuclear staining of DMT1 in COS-7 cells using a nonselective antibody to common epitopes on the N-terminal region for both isoforms of the transporter (Tabuchi et al., 2000). In contrast, when COS-7 cells were transfected with chimeric green fluorescent protein (GFP)-DMT1, labeling was detected in late endosomes but not in the nucleus. This result is again consistent with our findings, because the GFPDMT1 chimeric DNA contained the +IRE form of human DMT1, allowing one to infer that only the -IRE form is capable of accumulating within the nuclei. Interestingly, the nuclear signal is called p66, indicating that it has a size similar to that reported herein.

The different staining patterns observed for the two species of DMT1 are not artifacts, because peptides used to prepare the two antibodies were able to inhibit their respective signals selectively in the sympathetic neurons. In addition, a PSI-Blast search of the GenBank database for the specific - IRE peptide sequence used to generate the antibody returned only DMT1 as containing the relevant amino acid sequence. Specificity of the affinity-purified antibody preparations was also confirmed by the fact that only the -IRE antibody detected expression of a -IRE DMT1 construct after transient transfection of HEK293T cells. All of this evidence supports the conclusion that staining of the nucleus is, most likely, caused by the presence of the -IRE isoform of DMT1 in the nucleus in both PC12 cells and rat sympathetic neurons.

Colocalization experiments of the +IRE and -IRE forms of DMT1 with that of TfR demonstrate that these proteins generally do not colocalize extensively within the cells. TfR predominates in vesicles both within the cell body and in dendrites of rat sympathetic neurons. The +IRE isoform of DMT1 only partially colocalized with TfR in both the cell body and neurite extensions, although both proteins are excluded from the nucleus. The +IRE form of DMT1 distributed relatively uniformly within the cell body but not on the cell surface, whereas TfR is found preferentially on the cell surface and in perinuclear vesicles considered to be recycling endosomes (Mellman, 1996). The -IRE isoform of DMT1 and TfR also clearly display distinct distribution patterns in these neuronal cells. As noted above, most of the -IRE species appear in both the nucleus and the cell surface, including that of the dendrites and axons extensions. There is considerably less associated with the cell body, where it is not concentrated in perinuclear vesicles such as TfR. TfR, however, does appear to colocalize with the -IRE form of DMT1 on the cell surface, although TfR also appears on the surface independent of this species of DMT1. The modest colocalization of TfR observed with either species of DMT1 is somewhat surprising, because TfR is presumed to work in synchrony with DMT1 to facilitate transport of iron and other heavy metals into the cell. This implies that DMT1 may have different functions with regard to iron distribution throughout the cell. During the course of our studies, a paper appeared by Tabuchi et al. (2000) indicating that TfR and DMT1, detected by common epitopes on the N-terminal sequence, colocalize to only a modest extent in HEp2 cells, implying that DMT1 predominantly resides in the late endosomes.

Studies were also performed to see whether nuclear accumulation of the -IRE species would occur in the nucleus of other cell types maintained in culture. Staining in Schwann cells was diff use and observed in equal levels in both the cytoplasm and nucleus. These results indicate that the presence of the -IRE form of DMT1 in the nucleus of neurons is not simply dependent on culture conditions that often vary between cell lines. Thus, neuronal or neuronal-like cells appear preferentially to concentrate the -IRE form of DMT1 in the nucleus. Results with human HEK293T, HEP2G, and medulloblastoma cells also confirm that the two affinity-purified polyclonal antibody preparations can crossreact with human DMT1. The same unique subcellular distribution of the two isoforms of DMT1 observed with rat cells is also observed with human cells, confirming that this distribution is independent of mammalian species.

As noted above, Tabuchi et al. (2000) found DMT1 to associate primarily with late endosomes, although they did not distinguish between the two isoforms. Transfection studies similar to ours with epitope-tagged protein by $\mathrm{Su}$ et al. (1998) also failed to identify DMT1 in the nucleus of HEK293T cells. Similarly, Gruenheid et al. (1999), using anti-DMT1 that recognized epitope(s) common to both species of DMT1, detected a signal for both transferrin and DMT1 in endosomes but not in nuclei in Chinese hamster ovary cells, RAW cells, MEL cells, and TM4 cells. Subsequently, Trinder et al. (2000), using antibodies to a common epitope, noted that DMT1 is not found in nuclei of hepatic and gastrointestinal tissues. 
Canonne-Hergaux et al. (1999), also using antibodies to a common epitope, found that the transporter predominates near the apical surface of duodenal cells. These studies are consistent with the findings presented in this manuscript demonstrating the selective localization of the -IRE form of DMT1 in nuclei of neuronal cells or cells of neuronal origin. However, because we do not understand the mechanisms directing the transport of this protein to the nucleus, at this point we cannot exclude the possibility that other cells may also present a similar pattern of distribution for the -IRE species of DMT1. Thus, as noted above, the possibility that it may also occur in the nucleus of COS-7 cells deserves more investigation.

The concept of DMT1 has changed from its pre-1997 identity as a protein similar in sequence to Nramp1 (Gruenheid et al., 1995) to recognition of its role as a major duodenal ferrous iron transporter (Fleming et al., 1997; Gunshin et al., 1997, 1998) and exporter of ferrous iron (Garrick et al., 1993; Fleming et al., 1998) and other divalent metals (Gunshin et al., 1997) from endosomes as well as its role in the transferrin-independent uptake of iron (Hodgson et al., 1995; Garrick et al., 1999). We have found that the +IRE form of DMT1 localizes in neurons and other cells in a manner consistent with a role in iron uptake and possibly with a role in the uptake of other metals. In particular, the +IRE form is present on the cell surface and is within vesicles in dendrites and axons and the cell body in a distribution consistent with that of a subpopulation of endosomes but is not located in the nuclei. The -IRE form, however, is found in nuclei of neurons as well as on the cell surface, although it has a more expected distribution (i.e., not enriched in nuclei) in a variety of non-neuronal cells. This unexpected finding leads us to postulate that the two isoforms have different functions, at least in neurons. The nuclear localization of the -IRE form could imply that it (1) sequesters a divalent metal within nuclei, (2) transports divalent metals to nuclei, or (3) carries a signal (presumably relevant to metal homeostasis) to the nuclei. Studies are currently under way to characterize the structural components of this form of DMT1, investigating its nuclear localization and the role of this protein in regulating the entrance of divalent metals into the nucleus.

\section{REFERENCES}

Burdo JR, Martin J, Menzies SL, Dolan KG, Romano MA, Fletcher RJ, Garrick MD, Garrick LM, Connor JR (1999) Cellular distribution of iron in the brain of the Belgrade rat. Neuroscience 93:1189-1196.

Cameron PL, Sudhof TC, Jahn R, DeCamilli P (1991) Colocalization of synaptophysin with transferrin receptors: implications for synaptic vesicle biogenesis. J Cell Biol 115:151-164.

Canonne-Hergaux F, Gruenheid S, Ponka P, Gros P (1999) Cellular and subcellular localization of the Nramp2 iron transporter in the intestinal brush border and regulation by dietary iron. Blood 93:4406-4417.

Cassano S, Gallo A, Buccigrossi V, Porcellini A, Cerillo R, Gottesman ME, Avvedimento EV (1996) Membrane localization of cAMPdependent protein kinase amplifies cAMP signaling to the nucleus in PC12 cells. J Biol Chem 271:29870-29875.

Desole MS, Sciola L, Delogu MR, Sircana S, Migheli R (1996) Manganese and 1-methyl-4-(2'-ethylphenyl)-1,2,3,6-tetrahydropyridine induce apoptosis in PC12 cells. Neurosci Lett 209:193-196.

Desole MS, Esposito G, Migheli R, Sircana S, Delogu MR, Fresu L, Miele M, Denatale G, Miele E (1997a) Glutathione deficiency potentiates manganese toxicity in rat striatum and brainstem and in PC12 cells. Pharmacol Res 36:285-292.

Desole MS, Sciola L, Delogu MR, Sircana S, Migheli R, Miele E (1997b) Role of oxidative stress in the manganese and 1-methyl-4-(2'ethylphenyl)-1,2,3,6-tetrahydropyridine-induced apoptosis in PC12 cells. Neurochem Int 31:169-176

Fleming MD, Trenor CC, Su MA, Foernzler D, Beier DR, Dietrich WF, Andrews NC (1997) Microcytic anaemia mice have a mutation in Nramp2, a candidate iron transporter gene. Nat Genet 16:383-386.

Fleming MD, Romano MA, Su MA, Garrick LM, Garrick MD, Andrews NC (1998) Nramp2 is mutated in the anemic Belgrade $(b)$ rat: evidence of a role for Nramp2 in endosomal iron transport. Proc Natl Acad Sci USA 95:1148-1153.

Garrick MD, Dolan KG (2000) An expression system for a transporter of iron and other metals. In: Free radical and oxidant protocols, Vol 3 (Armstrong D, ed). Totawa, NJ: Humana, in press.

Garrick MD, Gniecko K, Liu Y, Cohan DS, Garrick LM (1993) Transferrin and the transferrin cycle in Belgrade rat reticulocytes. J Biol Chem 268:14867-14874.

Garrick LM, Dolan KG, Romano MA, Garrick MD (1999) Nontransferrin-bound iron uptake in Belgrade and normal rat erythroid cells. J Cell Physiol 178:349-358.

Gruenheid S, Cellier M, Vidal S, Gros P (1995) Identification and characterization of a second mouse Nramp gene. Genomics 25:514-525.

Gruenheid S, Canonne-Hergaux F, Gauthier S, Hackam DJ, Grinstein S, Gros P (1999) The iron transport protein NRAMP2 is an integral membrane glycoprotein that colocalizes with transferrin in recycling endosomes. J Exp Med 189:831-841.

Gunshin H, Mackenzie B, Berger UV, Gunshin Y, Romero MF, Boron WF, Nussberger S, Gollan JL, Hediger MA (1997) Cloning and characterization of a mammalian proton-coupled metal-ion transporter. Nature 388:482-488.

Gunshin H, Rouault T, Rogers J, Allerson C, Gollan JL, Hediger MA (1998) Regulation of the divalent cation transporter DCT1 at the mRNA level. FASEB J 12:A820.

Haile DJ (1999) Regulation of genes of iron metabolism by the ironresponse proteins. Am J Med Sci 318:230-240.

Higgins D, Lein PJ, Osterhout DO, Johnson MI (1991) Tissue culture of mammalian autonomic neurons. In: Culturing nerve cells (Banker G, Goslin K, eds), pp 177-206. Cambridge, MA: MIT.

Hirata Y, Adachi K, Kiuchi K (1998) Activation of PNK pathway and induction of apoptosis by manganese in PC12 cells. J Neurochem 71:1607-1615.

Hodgson LL, Quail EA, Morgan EH (1995) Iron transport mechanisms in reticulocytes and mature erythrocytes. J Cell Physiol 162:181-190.

Kim HD, Sun GY, Sun AY (1993) Manganese transport and $\mathrm{Na} / \mathrm{K} / \mathrm{Cl}$ cotransport in PC-12 cells. Ann NY Acad Sci 679:376-381.

Lee PL, Gelbart T, West C, Halloran C, Beutler E (1998) The human Nramp2 gene: characterization of the gene structure, alternative splicing, promoter region and polymorphisms. Blood Cells Mol Dis 24:199-215.

Lein P, Johnson M, Guo X, Rueger D, Higgins D (1995) Osteogenic protein-1 induced dendritic growth in rat sympathetic neurons. Neuron 15:597-605.

Lin W, Higgins D, Pacheco M, Aletta J, Perini S, Marcucci KA, Rabin RA, Roth JA (1993) Manganese induces spreading and neurite process outgrowth in rat pheochromocytoma. J Neurosci Res 34:546-561.

Mellman I (1996) Endocytosis and molecular sorting. Annu Rev Cell Dev Biol 12:575-625.

Ponka P (1997) Tissue-specific regulation of iron metabolism and heme synthesis: distinct control mechanisms in erythroid cells. Blood 89:1-18.

Roth JA, Feng L, Walowitz J, Browne RW (2000) Manganese-induced rat pheochromocytoma (PC12) cell death is independent of caspase activation. J Neurosci Res 61:162-171.

Rouault T (1999) Targeted disruption of iron regulatory protein 2 in mice leads to neuronal iron overload and progressive neurodegeneration. Blood 94[Suppl 1]:56.

Rouault T, Klausner R (1997) Regulation of iron metabolism in eukaryotes. Curr Top Cell Regul 35:1-19.

Schrantz N, Blanchard DA, Mitenne F, Auffredou M-T, Vazquez A, Leca G (1999) Manganese induces apoptosis of human B cells: caspasedependent cell death blocked by Bcl-2. Cell Death Differ 6:445-453.

Schümann K, Moret R, Künzle H, Kühn LC (1999) Iron regulatory protein as an endogenous sensor of iron in rat intestinal mucosa: possible implications for the regulation of iron absorption. Eur J Biochem 260:362-372.

Su MA, Trenor CC, Fleming JC, Fleming MD, Andrews NC (1998) The G185R mutation disrupts function of the iron transporter Nramp2. Blood 92:2157-2163.

Tabuchi M, Yoshimori T, Yamaguchi K, Yoshida T, Kishi F (2000) Human NRAMP2/DMT1, which mediates iron transport across endosomal membranes, is localized to late endosomes and lysosomes in Hep-2 cells. J Biol Chem 275:22220-22228.

Trinder D, Oates PS, Thomas C, Sadleir J, Morgan EH (2000) Localisation of divalent metal transporter I (DMT1) to the microvillus membrane of rat duodenal enterocytes in iron deficiency, but to hepatocytes in iron overload. Gut 46:270-276.

Yanagiya T, Imura N, Enomoto S, Kondo Y, Himeno S (2000) Suppression of a high-affinity transport system for manganese in cadmiumresistant metallothionein-null cells. J Pharmacol Exp Ther 292:10801086. 